\title{
Hybrid Filtered-x Adaptive Vibration Control with Internal Feedback and Online Identification
}

\author{
Lihua Yang $\mathbb{D}^{1},{ }^{1}$ Shuyong Liu, ${ }^{2}$ Haipeng Zhang, ${ }^{1}$ Haiping Wu, ${ }^{1}$ Haifeng Li, ${ }^{1}$ and Jian Jiang ${ }^{3}$ \\ ${ }^{1}$ Power Control Department, Navy Submarine Academy, Qingdao 266199, China \\ ${ }^{2}$ College of Power Engineering, Naval University of Engineering, Wuhan 430033, China \\ ${ }^{3}$ Institute of Acoustics, Chinese Academy of Sciences, Beijing 100190, China
}

Correspondence should be addressed to Lihua Yang; dreamfly4@163.com

Received 22 October 2017; Revised 4 March 2018; Accepted 25 April 2018; Published 18 July 2018

Academic Editor: Brij N. Agrawal

Copyright (C) 2018 Lihua Yang et al. This is an open access article distributed under the Creative Commons Attribution License, which permits unrestricted use, distribution, and reproduction in any medium, provided the original work is properly cited.

Active control is an effective way to suppress low-frequency mechanical vibration. However, with applications to submarine equipment, there are still some shortcomings due to vibration coupling and multifrequency complex excitation. In this paper, a novel hybrid improved adaptive control strategy, feedback and online identification filtered-x LMS, namely, FOFxlms, is proposed, which introduces the residual errors to correct variable step-size, uses the estimated primary path to improve online identification, and applies internal feedback to compensate for the feedforward control. Then the FOFxlms algorithm is applied to a double-layer vibration isolation system of submarine rotating equipment, and the simulation results show that the normalized variable step-size with residual error can effectively improve convergence speed, the internal feedback can efficaciously compensate for steady-state control accuracy, and the online identification can dynamically identify the time-varying characteristics of the secondary path. The vibration reduction efficiency of Fxlms, FFxlms, and FOFxlms increases for the fundamental frequency vibration; the control effect and convergence speed are also enhanced in turn.

\section{Introduction}

Mechanical vibration makes great contribution to submarine radiation noise, and the vibration isolation, mainly including passive and active vibration isolation, is an effective way to control the vibration transfer. As is known to all, the passive vibration isolation systems are easily installed and do not require external energy. They have been widely applied to the vibration control of ship machinery and equipment and have achieved good control effect in medium and high frequency bands along with the development of passive vibration isolation such as single layer, double layer, and floating raft [1]. However, passive vibration isolation still has some deficiencies such as the contradiction between low dynamic stiffness and instability and the narrower vibration isolation band. In addition, the excitation frequency deviates from the effective isolation band due to many complex conditions with mechanical equipment. Passive vibration isolation devices cannot adapt to the system changes for their fixed parameters, which may lead to the deterioration of control effect. Therefore, it is still difficult to achieve a more effective result in the low-frequency bands.

Studies have shown that active control can be used to control low-frequency vibration effectively [2] and has been applied to precision equipment manufacturing, civil engineering, mechanical vibration control in ship, and so on. The new "Sea Wolf" class nuclear submarine, equipped with active control system, has a good acoustic stealth performance [3]. A narrowband active absorption technology has been carried out on the floating raft in mine sweepers and achieved a better noise reduction as well. Winberg et al. [4] conducted a consultancy project on active vibration isolation in shipboard. Paulstra Vibrachoc company has also developed an active control system for large-scale equipment such as diesel engine [5]. In addition, the Institute of Sound and Vibration Research, Adelaide University, and others have achieved many fruitful research results as well $[6,7]$. The active control technology mainly includes actuators and control strategies; the latter is the core and also a hot and difficult research topic $[8,9]$. 
The Fxlms and its improvement algorithms have extensive applications in engineering [10]. Carra et al. [11], Ma et al. [12], Carnahan et al. [13], Zhu et al. [14], and Li et al. [15] have researched various Fxlms algorithms with applications to rectangular aluminum structures, piezoelectric cantilever structures, and gear transmission systems and analyzed the relationship between the convergence of Fxlms and Fulms algorithms and control path. Das et al. [16] proposed a frequency domain Fxlms algorithm with high efficiency. Huang et al. [17], Kim et al. [18], Ning et al. [19], and Zhang et al. [20] got single-related improved Fxlms and Fulms algorithms to compare the calculation efficiency and control effect with traditional ones. Although the above researches have done much work in adaptive algorithm improvement and its applications, there are still some shortcomings in practice such as the active control mainly for single frequency excitation; the contradiction between control precision and convergence speed for fixed-step-size algorithms; more dependence between convergence rate and autocorrelation matrix of input signals; and much weaker adaptive ability for time-varying system of offline identification. In this regard, Akhtar et al. [21], Carini et al. [22], Chan et al. [23], Pu et al. [24], Avalos et al. [25], and other scholars have conducted researches to improve the single-related issue by limiting secondary path to avoid nonlinear distortion, by using variable step-size to increase convergence speed, and by applying online identification to control time-varying path $[26,27]$.

In submarine, the parameters of passive isolation systems are often time-varying with the rigid-flexible coupling and complex excitation. In order to improve the control effect, it is necessary to carry out the paths interaction and online identification at the same time. In addition, the actuators, generally installed in parallel with the isolators, also produce an active control force between the primary and secondary paths synchronously, forming a feedback loop. Nevertheless, the traditional Fxlms algorithm only considers its effect on the latter and ignores the influence on the reference signal of the former, which may lead to the reference signal instability and affect convergence speed and control precision [28].

To address these special needs for active vibration isolation systems in submarine, a novel hybrid adaptive algorithm is proposed in this paper, which combines variable step-size, online identification, internal feedback, and other factors.

\section{Double-Layer Isolation System of Submarine Equipment}

In this paper, a double-layer vibration isolation platform with rotating equipment is taken as the study object, and its Adams virtual prototype, as shown in Figure 1, can be simplified as Figure 2 which is established in the case of ignoring the motion of torsion, rolling, and only considering vertical vibration.

In Figures 1 and 2, $M_{1}, K_{1}$, and $C_{1}$ are the mass of upper rotating machinery, vibration isolator stiffness, and damping, respectively. $M_{2}, K_{2}$, and $C_{2}$ represent the mass of the middle platform, vibration isolator stiffness, and damping, respectively. $f_{0}, f$ are the external excitation and active

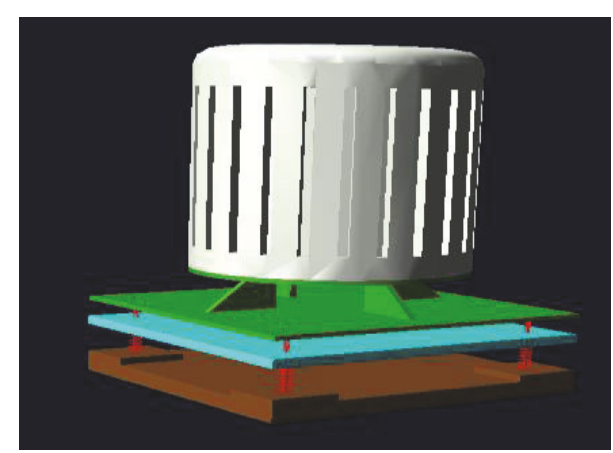

FIGURE 1: Vibration platform virtual prototype with rotating equipment.

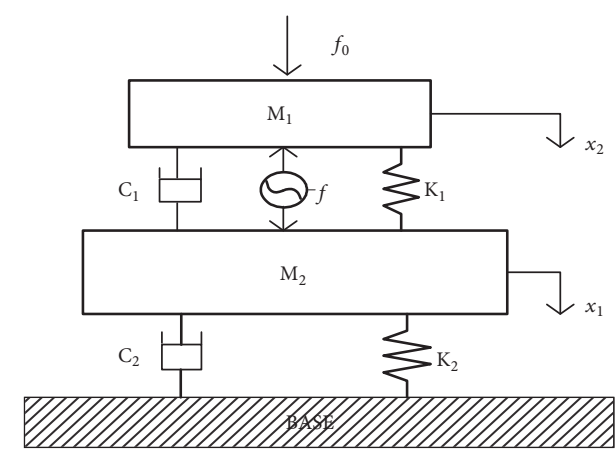

FIGURE 2: Simplified model of vibration platform virtual prototype.

control force, respectively. $x_{2}, \dot{x}_{2}, \ddot{x}_{2}, x_{1}, \dot{x}_{1}$, and $\ddot{x}_{1}$ denote the displacements, velocities, and acceleration of the upper equipment and middle platform, respectively. Then, the system dynamic equations can be expressed as follows:

$$
\begin{aligned}
& M_{1} \ddot{x}_{2}+C_{1}\left(\dot{x}_{2}-\dot{x}_{1}\right)+K_{1}\left(x_{2}-x_{1}\right)=f_{0}-f \\
& M_{2} \dot{x}_{1}+C_{2} \dot{x}_{1}+K_{2} x_{1}+C_{1}\left(\dot{x}_{1}-\dot{x}_{2}\right)+K_{1}\left(x_{1}-x_{2}\right) \\
& \quad=f .
\end{aligned}
$$

The Laplace transformation of (1) can be obtained as below:

$$
\begin{aligned}
& M_{1} S^{2} X_{2}+C_{1} S\left(X_{2}-X_{1}\right)+K_{1}\left(X_{2}-X_{1}\right)=F_{0 s}-F_{s} \\
& M_{2} S^{2} X_{1}+C_{2} S X_{1}+K_{2} X_{1}+C_{1} S\left(X_{1}-X_{2}\right) \\
& \quad+K_{1}\left(X_{1}-X_{2}\right)=F_{s} .
\end{aligned}
$$

Transform (2) and eliminate the variable $X_{2}$ :

$$
\begin{gathered}
\left(M_{1} M_{2} S^{4}+\left(M_{1} C_{1}+M_{1} C_{2}+M_{2} C_{1}\right) S^{3}\right. \\
+\left(M_{1} K_{1}+M_{1} K_{2}+M_{2} K_{1}+C_{1} C_{2}\right) S^{2}
\end{gathered}
$$




$$
\begin{aligned}
& \left.+\left(C_{1} K_{2}+C_{2} K_{1}\right) S+K_{1} K_{2}\right) X_{1}=M_{1} S^{2} F_{s}+\left(C_{1} S\right. \\
& \left.+K_{1}\right) F_{0 s} .
\end{aligned}
$$

Let $F_{0 s}=0$ and $F_{s}=0$ in turn; the transfer function can be obtained between the displacement $x_{1}$ and active control force $f$ or excitation force $f_{0}$, respectively:

$$
\begin{aligned}
& \frac{X_{1}}{F_{s}}=\frac{C_{1} S+K_{1}}{M_{1} M_{2} S^{4}+\left(M_{1} C_{1}+M_{1} C_{2}+M_{2} C_{1}\right) S^{3}+\left(M_{1} K_{1}+M_{1} K_{2}+M_{2} K_{1}+C_{1} C_{2}\right) S^{2}+\left(C_{1} K_{2}+C_{2} K_{1}\right) S+K_{1} K_{2}} \\
& \frac{X_{1}}{F_{0 s}}=\frac{M_{1} S^{2}}{M_{1} M_{2} S^{4}+\left(M_{1} C_{1}+M_{1} C_{2}+M_{2} C_{1}\right) S^{3}+\left(M_{1} K_{1}+M_{1} K_{2}+M_{2} K_{1}+C_{1} C_{2}\right) S^{2}+\left(C_{1} K_{2}+C_{2} K_{1}\right) S+K_{1} K_{2}} .
\end{aligned}
$$

Transform (2) and eliminate the variable $X_{1}$ :

$$
\begin{aligned}
& \left(M_{1} M_{2} S^{4}+\left(M_{1} C_{1}+M_{1} C_{2}+M_{2} C_{1}\right) S^{3}\right. \\
& +\left(M_{1} K_{1}+M_{1} K_{2}+M_{2} K_{1}+C_{1} C_{2}\right) S^{2} \\
& \left.+\left(C_{1} K_{2}+C_{2} K_{1}\right) S+K_{1} K_{2}\right) X_{2}=\left(M_{2} S^{2}\right.
\end{aligned}
$$

$$
\begin{aligned}
& \left.+\left(C_{1}+C_{2}\right) S+K_{1}+K_{2}\right) F_{s}-\left(M_{2} S^{2}+C_{2} S+K_{2}\right) \\
& \cdot F_{0 s} \cdot
\end{aligned}
$$

Let $F_{0 s}=0$ and $F_{s}=0$ in turn; the transfer function can be also obtained between the displacement $x_{2}$ and active control force $f$ or excitation force $f_{0}$, respectively:

$$
\begin{aligned}
& \frac{X_{2}}{F_{s}}=\frac{M_{2} S^{2}+\left(C_{1}+C_{2}\right) S+K_{1}+K_{2}}{M_{1} M_{2} S^{4}+\left(M_{1} C_{1}+M_{1} C_{2}+M_{2} C_{1}\right) S^{3}+\left(M_{1} K_{1}+M_{1} K_{2}+M_{2} K_{1}+C_{1} C_{2}\right) S^{2}+\left(C_{1} K_{2}+C_{2} K_{1}\right) S+K_{1} K_{2}} \\
& \frac{X_{2}}{F_{0 s}}=\frac{-M_{2} S^{2}-C_{2} S-K_{2}}{M_{1} M_{2} S^{4}+\left(M_{1} C_{1}+M_{1} C_{2}+M_{2} C_{1}\right) S^{3}+\left(M_{1} K_{1}+M_{1} K_{2}+M_{2} K_{1}+C_{1} C_{2}\right) S^{2}+\left(C_{1} K_{2}+C_{2} K_{1}\right) S+K_{1} K_{2}} .
\end{aligned}
$$

Let $a=M_{1} M_{2}, b=M_{1} C_{1}+M_{1} C_{2}+M_{2} C_{1}, c=M_{1} K_{1}+$ $M_{1} K_{2}+M_{2} K_{1}+C_{1} C_{2}, d=C_{1} K_{2}+C_{2} K_{1}, e=K_{1} K_{2}$; then the transfer function between the acceleration and excitation force or active control force can be rewritten as

$$
\begin{aligned}
& P_{(\mathrm{s})}=\frac{X_{1}}{F_{0 s}} S^{2}=\frac{M_{1} S^{4}}{a S^{4}+b S^{3}+c S^{2}+d S+e} \\
& S_{(\mathrm{s})}=\frac{X_{1}}{F_{s}} S^{2}=\frac{\left(C_{1} S+K_{1}\right) S^{2}}{a S^{4}+b S^{3}+c S^{2}+d S+e} \\
& Q_{(\mathrm{s})}=\frac{X_{2}}{F_{s}} S^{2}=\frac{\left(M_{2} S^{2}+\left(C_{1}+C_{2}\right) S+K_{1}+K_{2}\right) S^{2}}{a S^{4}+b S^{3}+c S^{2}+d S+e} \\
& G_{(\mathrm{s})}=\frac{X_{2}}{F_{0 s}} S^{2}=\frac{-\left(M_{2} S^{2}+C_{2} S+K_{2}\right) S^{2}}{a S^{4}+b S^{3}+c S^{2}+d S+e} .
\end{aligned}
$$

$P(s)$ is the transfer function, between the acceleration of the middle platform and the exciting force of upper mass, and it is called the primary path. $S(s)$ is the transfer function, between the acceleration of the middle platform and active control force, and it can be referred to as the secondary path including power amplifiers and actuators. $G(s)$ and $Q(s)$ are the transfer functions between the upper acceleration and the excitation force or active control force, respectively, and the latter can be called the internal feedback path.

\section{Adaptive LMS and Fxlms Algorithms}

3.1. LMS Algorithm. In general, adaptive filtering algorithm can be mainly divided into RLS and LMS algorithm according to the optimization criteria. Compared to RLS, LMS algorithm, proposed by Hoff and Widrow in 1960s [29], has advantages of easier implement and stronger robustness and also has been widely used in noise cancellation, system identification, signal filtering, and related fields [30].

LMS algorithm is shown in Figure 3, where $W(n), d(n)$, $x(n), y(n)$, and $e(n)$ denote the horizontal FIR filter, the reference input signal, the filter input signal, the filter output signal, and the error signal, respectively. The input signal time series $X(n)$ and filter coefficients $W(n)$ can be expressed as follows:

$$
\begin{aligned}
X(n) & =[x(n), x(n-1), \ldots, x(n-N+1)]^{T} \\
W(n) & =\left[w_{0}(n), w_{1}(n), \ldots, w_{N-1}(n)\right]^{T} .
\end{aligned}
$$

Then, the filter output $y(n)$ and the system error $e(n)$ are given by

$$
\begin{aligned}
y(n) & =\sum_{i=0}^{N-1} w_{i} x(n-i)=W^{T}(n) X(n) \\
& =X^{T}(n) W(n) . \\
e(n) & =d(n)-y(n)=d(n)-W^{T}(n) X(n)
\end{aligned}
$$




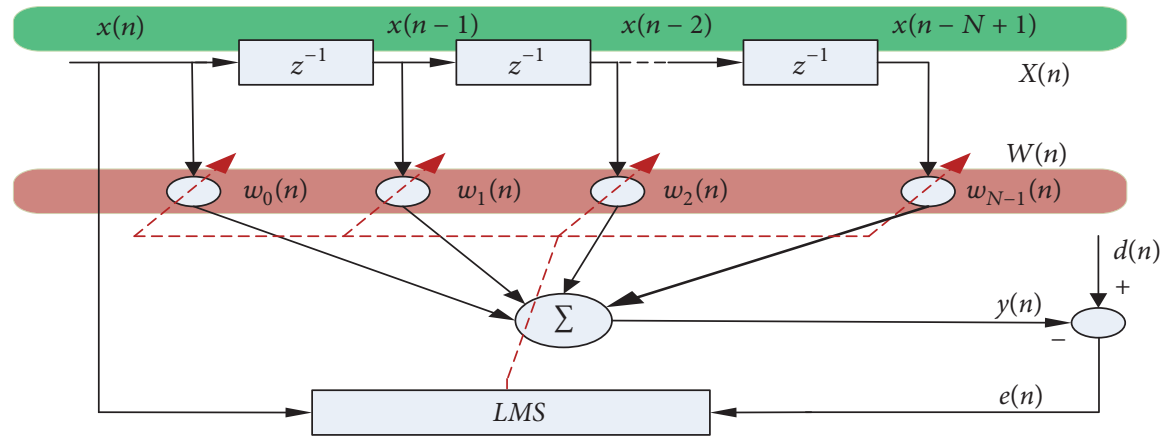

FIGURE 3: LMS algorithm structure.

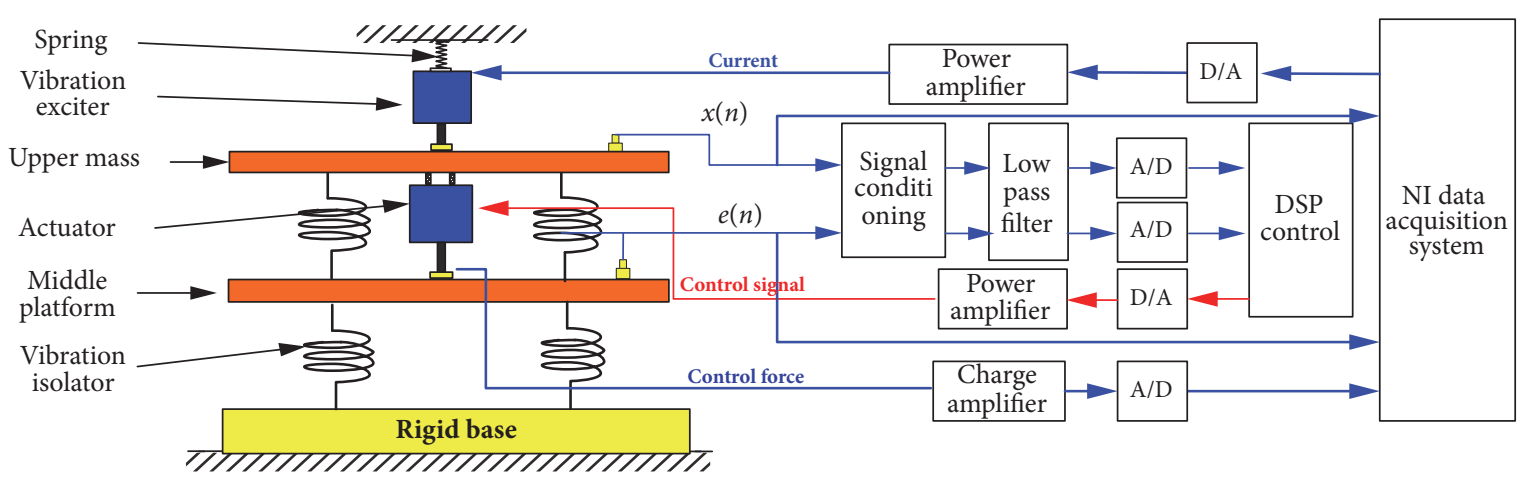

FIgURE 4: Active vibration control system.

Here, using instantaneous gradient instead of real gradient, the filter coefficients $W(n+1)$ will infinitely approach the Wiener solution $W(n)^{*}$ by the steepest descent method, and the LMS algorithm vector coefficients can be obtained as

$$
W(n+1)=W(n)+\mu e(n) X(n) .
$$

The LMS algorithm flow is calculated as follows.

Step 1. Initialize filter coefficient $W_{M}=\left[\begin{array}{llll}0 & 0 & \cdots & 0\end{array}\right]^{T}$.

Step 2. Update time series $n=1,2, \cdots$, and calculate filter coefficients in (17).

Step 3. Update filter output $y(n)$ and error output $e(n)$ in (15) and (16), respectively.

3.2. Filtered- $x$ LMS Algorithm. The LMS algorithm has been briefly introduced in Section 3.1. While applying LMS to vibration control systems, the influence of the secondary path including power amplifiers and actuators should be considered. The experimental principle and test platform are shown in Figure 4, which corresponds to the structure of adaptive control algorithm for the single input and single output (SISO) system in Figure 5.

It can be seen from Figures 4 and 5 that control principle can be expressed as follows: Firstly, the upper acceleration or excitation force $x(n)$ and middle acceleration signal $e(n)$ are collected by sensors. The signals are then transferred to DSP controller by A/D conversion, which can generate an output

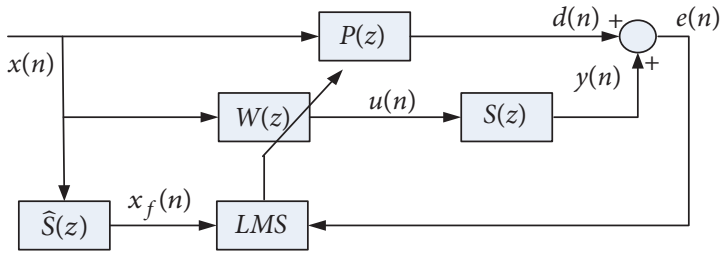

Figure 5: Adaptive filter algorithm structure.

control signal $u(n)$ by calculation of controller $W(z)$ and D/A conversion. Furthermore, the interactive force, located between the upper and middle layers, can be produced by the secondary path $S(z)$ including power amplifiers and actuators. It will generate a secondary output $y(n)$ with equal magnitude and opposite phase to the vibration signal $d(n)$ at the same time. Finally, the goal of reducing $d(n)$ can be achieved by superposition in the middle layer. The physical meanings of relevant variables in Figure 5 are as follows:

$\widehat{S}(z)$ is the estimated value of the secondary path $S(z)$, and $x(n)$ is the upper excitation, it is commonly known as the reference signal. $u(n)$ is the filter output signal, and $x_{f}(n)$ is the filtered signal of $x(n) . d(n)$ is the middle acceleration induced by the primary path $P(z), y(n)$ is the acceleration produced by the secondary path $S(z)$. $e(n)$ is the residual signal. $W(z)$ is the FIR weight vector of the controller. When $S(z)$ and its estimated value $\widehat{S}(z)$ tend to be the same as each other, Figure 5 will degenerate into the algorithm described 
in Figure 3. As shown in Figure 5, $e(n)$ is the sum of $d(n)$ and $y(n)$, so it can be given by

$$
e(n)=d(n)+y(n) .
$$

$W(z)$ is $N$-order FIR filter, and the output $u(n)$ can be expressed as convolution operation:

$$
\begin{aligned}
u(n) & =\sum_{i=0}^{N-1} w_{i}(n) x(n-i)=W^{T}(\mathrm{n}) X(n) \\
& =X^{T}(n) W(n)
\end{aligned}
$$

where $X(n), W(n)$ are the delay sampling sequence and the filter weight vector at the moment $n$, respectively.

$$
\begin{aligned}
X(n) & =\left[\begin{array}{ll}
x(n) & x(n-1) \ldots x(n-(N-1))
\end{array}\right]^{T} \\
W(n) & =\left[\begin{array}{lll}
w_{0}(n) & w_{1}(n) \ldots w_{N-1}(n)
\end{array}\right]^{T} .
\end{aligned}
$$

When $S(z)$ can also be replaced by a FIR filter, its weight coefficients will be described as below:

$$
S(n)=\left[\begin{array}{llll}
s_{0}(n) & s_{1}(n) & \ldots & s_{M-1}(n)
\end{array}\right]^{T}
$$

where $s_{i}(n)$ is the $i$-th impulse response coefficient of $S(n)$ at the moment $n$, and its output $y(n)$ is defined as

$$
\begin{aligned}
y(n) & =\sum_{i=0}^{M-1} s_{i}(n) u(n-i)=S(n)^{T} U(n) \\
& =U(n)^{T} S(n)
\end{aligned}
$$

where $U(n)$ is the $M \times 1$ order vector sequence which consists of the output $u(n)$ of the filter $W(n)$.

$$
U(n)=[u(n) u(n-1) \ldots u(n-M+1)]^{T}
$$

Substituting (19) and (24) into (23), we can get the secondary path output $y(n)$ as

$$
\begin{aligned}
y(n) & =\left[X^{T}(n) W(n)\right]^{T} S(n)=W^{T}(n) X(n) S(n) \\
& =W^{T}(n) X_{f}(n)=X_{f}^{T}(n) W(n)
\end{aligned}
$$

where $X_{f}(n)$ is the filtered reference signal $X(n)$ filtered by estimated secondary path $\widehat{S}(z)$.

$$
\begin{aligned}
& X_{f}(n)=\left[\begin{array}{ll}
x_{f}(n) & x_{f}(n-2) \ldots x_{f}(n-N+1)
\end{array}\right]^{T} \\
& x_{f}(n)=\sum_{i=0}^{M-1} s_{i}(n) x(n-i)
\end{aligned}
$$

The active control is to minimize residual signal $e(n)$, so the objective function can be defined as[29]

$$
J(n)=E\left[e^{2}(n)\right]
$$

Commonly, the expected value $E\left(e^{2}(n)\right)$ can be replaced by instantaneous error $e^{2}(n)$.

$$
\begin{aligned}
J(n) & \approx e^{2}(n)=\left(d(n)+\sum_{i=0}^{M-1} s_{i}(n) u(n-i)\right)^{2} \\
& =\left(d(n)+\sum_{i=0}^{M-1} s_{i}(n) \sum_{i=0}^{N-1} w_{j}(n-i) x(n-i-j)\right)^{2} .
\end{aligned}
$$

Then, the instantaneous gradient estimation of the objective function $J(n)$ can be expressed as

$$
\begin{aligned}
\frac{\partial J(n)}{\partial W(n)} & =2 e(n) \frac{\partial e(n)}{\partial y(n)} \frac{\partial y(n)}{\partial W(n)} \\
& =2 e(n) \sum_{i=0}^{M-1} s_{i}(n) \frac{\partial u(n-i)}{\partial W(n)} \\
& =2 e(n) \sum_{i=0}^{M-1} s_{i}(n)\left[\begin{array}{c}
\frac{\partial u(n-i)}{\partial w_{0}(n)} \\
\frac{\partial u(n-i)}{\partial w_{1}(n)} \\
\vdots \\
\frac{\partial u(n-i)}{\partial w_{N-1}(n)}
\end{array}\right] .
\end{aligned}
$$

Assume that the iterative step-size is very small, and the filter weight coefficient is relatively slow to update in a smaller period.

$$
\frac{\partial u(n-i)}{\partial w_{j}(n)} \approx \frac{\partial u(n-i)}{\partial w_{j}(n-i)}, \quad j=0,1, \ldots, N-1
$$

Thus, (30) can be rewritten as

$$
\begin{gathered}
\frac{\partial J(n)}{\partial W(n)} \approx 2 e(n) \sum_{i=0}^{M-1} s_{i}(n)\left[\begin{array}{c}
\frac{\partial u(n-i)}{\partial w_{0}(n-i)} \\
\frac{\partial u(n-i)}{\partial w_{1}(n-i)} \\
\vdots \\
\frac{\partial u(n-i)}{\partial w_{N-1}(n-i)}
\end{array}\right] \\
=2 e(n)\left[\begin{array}{c}
\sum_{i=0}^{M-1} s_{i}(n) x(n-i) \\
\sum_{i=0}^{M-1} s_{i}(n) x(n-i-1) \\
\vdots \\
\sum_{i=0}^{M-1} s_{i}(n) x(n-i-N+1)
\end{array}\right] .
\end{gathered}
$$

If the secondary path does not change with time, substituting (26) and (27) into (32), we can get

$$
\frac{\partial J(n)}{\partial W(n)}=2 e(n) X_{f}(n) \text {. }
$$


Then, the weight of filtered adaptive control algorithm can be described by

$$
W(n+1)=W(n)-\mu e(n) X_{f}(n)
$$

where the iterative step-size $\mu$ has a great influence on the convergence speed. Compared with (17) and (34), it is known that $X_{f}(n)$ replaces reference input $X(n)$. Therefore, this algorithm is called the Fxlms, and the calculation steps can be expressed as follows.

Step 1. Obtain the initial reference signal $x(n)$ and error signal $e(n)$.

Step 2. Calculate the control filter output signal $u(n)$ in (19).

Step 3. Calculate the output signal $y(n)$ of the secondary path in (25).

Step 4. Calculate the residual signal $e(n)$ and the filtered signal $x_{f}(n)$ in (18) and (27), respectively.

Step 5. Update the control filter $W(n)$ in (34).

\section{Hybrid Filtered-x LMS Algorithm}

Fxlms algorithm has been used in active vibration control for its high computational efficiency and strong adaptability [31]. However, the algorithm also has some deficiencies. To address these shortcomings, multiple factors will be carried out on the basis of Fxlms algorithm in this section.

4.1. Nonlinear Distortion. To limit the output power of the secondary path and avoid nonlinear distortion, a forgetting factor $\beta$ is introduced (its value is slightly less than one), and then the updating weight in (34) can be rewritten as

$$
W(n+1)=\beta W(n)-\mu e(n) X_{f}(n) .
$$

The Fxlms is an improvement of LMS algorithm, so the filter vector is not necessarily the optimal weight vector (Wiener solution) at the end of convergence, which may affect convergence speed and control effect. In order to overcome the fixed-step shortcomings, the normalized LMS algorithm and transient step-size LMS algorithm have been proposed [21], and the former is formulated as

$$
\mu(n)=\frac{\mu_{0}}{X^{T}(n) X(n)+e p s}
$$

where $\mu_{0}$ is the initial fixed-step, and a small positive number eps can prevent the system from divergence. However, this improvement does not reflect the change of the residual error. Hence, a further improvement is carried out by introducing the residual error; (36) will be revised as below:

$$
\mu(n)=\frac{\mu_{0}}{X^{T}(n) X(n)+E(n)+e p s}
$$

where $E(n)=\beta E(n-1)+(1-\beta) e^{2}(n)$ is residual error energy [32]. Equation (37) shows that the larger the reference signal

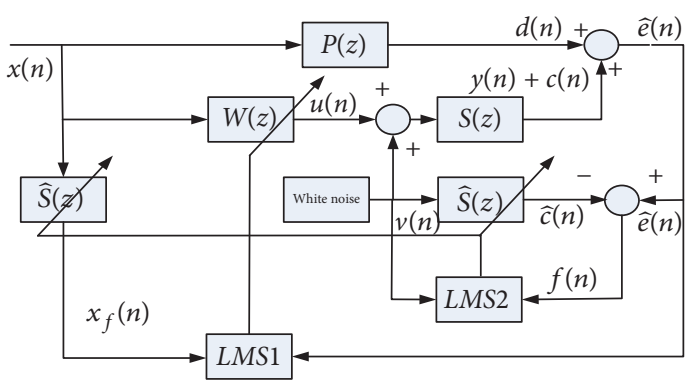

FIGURE 6: Online identification of secondary paths in Eriksson model.

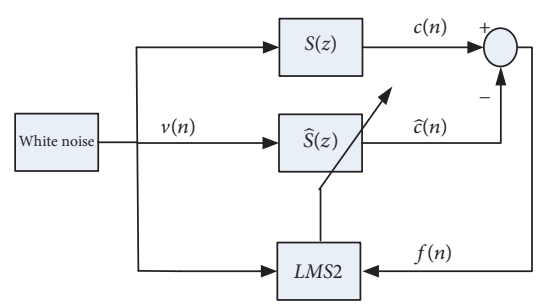

FIGURE 7: Offline identification model of secondary path.

or residual error, the smaller the step-size. This improvement can effectively solve the effect on control accuracy at the end of fixed-step convergence. While in initial state $X^{T}(n) X(n) \longrightarrow 0, E(n) \longrightarrow 0$, so $\mu(n)$ may be reaching a larger positive value. Here, in order to prevent a larger initial variable step-size making system divergence, it is still needed to limit the variable step-size, where the function $\operatorname{sat}(\mu(n))$ can be used to constrain the amplitude of variable step-size in (37).

4.2. Online Identification. When physical vibration isolation systems are relatively stable, the offline identification algorithm, shown in Figure 5, has the advantages of smaller computation, faster convergence speed, and better control effect. However, under the conditions of long time variable amplitude and frequency, the dynamics of the secondary path, including the power amplifiers, actuators, and other components, will inevitably be affected by factors such as temperature and deformation [23]. In this case, offline secondary path will not meet requirements of adaptive control stability and precision, so it is very important to study online identification. Figure 6, firstly proposed by Eriksson [33], shows the online identification of the secondary path.

Compared with Figure 5, the controller in Figure 6 is made up of two adaptive FIR filters, and white noise signal $v(n)$ with zero mean and variance $\sigma^{2}$ is introduced into the secondary path. This signal is superimposed with the control signal $u(n)$ to drive the secondary path $S(z)$, and the secondary path output is then changed from $y(n)$ to $y(n)+$ $c(n)$. The former part is the response of the original control signal $u(n)$, and the latter is the one caused by white noise $v(n)$. When the external excitation is zero, Figure 6 will be degraded to the offline identification shown in Figure 7. 


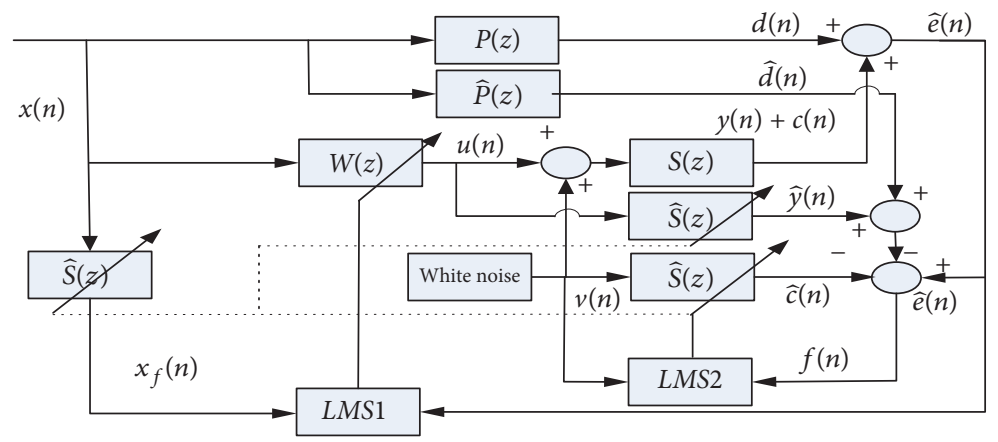

FIGURE 8: Improved online identification model of secondary paths.

According to the analysis above, the Fxlms with $S(z)$ online identification can be expressed as follows.

Step 1. Define residual signal $e(n), y(n)$, and $d(n)$ of original online identification in convolution form.

$$
\begin{aligned}
e(n) & =d(n)+y(n)=p(n) * x(n)+s(n) * u(n) \\
& =[p(n)+s(n) * w(n)] * x(n)
\end{aligned}
$$

Step 2. The white noise $v(n)$ and the control signal $u(n)$ are superimposed to drive the secondary path $S(z)$, and the former is also the reference input signal of the estimated secondary path $\widehat{S}(z)$ and $L M S 2$ algorithm.

Step 3. Calculate output signal $\widehat{c}(n)$ of the estimated secondary path $\widehat{S}(z)$.

$$
\widehat{c}(n)=\sum_{i=0}^{M-1} s_{i}(n) v(n-i)
$$

Step 4. Add the white noise excitation $v(n)$, and calculate the residual signal $\widehat{e}(n)$ of online identification.

$$
\begin{aligned}
\widehat{e}(n) & =d(n)+y(n)+c(n) \\
& =p(n) * x(n)+s(n) * u(n)+s(n) * v(n) \\
& =[p(n)+s(n) * w(n)] * x(n)+s(n) * v(n)
\end{aligned}
$$

If $v(n)$ and $x(n)$ are not related to each other, $\widehat{e}(n)$ may not affect its stability as the LMS1 input signal, but it will reduce the convergence speed.

Step 5. Update the weight coefficients of the adaptive filter LMS2.

$$
\begin{aligned}
w^{\prime}(n+1)= & w^{\prime}(n)+\mu^{\prime} f(n) v(n) \\
= & w^{\prime}(n) \\
& +\mu^{\prime}[d(n)+y(n)+c(n)-\widehat{c}(n)] v(n) \\
= & w^{\prime}(n)+\mu^{\prime}[c(n)-\widehat{c}(n)] v(n) \\
& +\mu^{\prime}[d(n)+y(n)] v(n)
\end{aligned}
$$

As is shown in (41), if $d(n)+y(n)$ is irrelevant to $v(n)$, $w^{\prime}(n)$ will converge to the optimal state and finally be stable, but this convergence process may be much slower due to the existence of $d(n)+y(n)$. In practical application, affected by external disturbance and sensor measurement error, it is inevitable that $d(n)$ and $y(n)$ have a correlation with $v(n)$, which may have influence on convergence speed and its stability. Therefore, a method, shown in Figure 8, is proposed to minimize $\mu^{\prime}[d(n)+y(n)] v(n)$. Here, one offline estimated primary path $\widehat{P}(z)$ and two online estimated secondary paths $\widehat{S}(z)$ are introduced, so the weight coefficient of the improved online identification can be updated as

$$
\begin{aligned}
w^{\prime} & (n+1)=w^{\prime}(n)+\mu^{\prime} f(n) v(n)=w^{\prime}(n) \\
+ & \mu^{\prime}[d(n)+y(n)+c(n)-\widehat{c}(n)-\widehat{y}(n)-\widehat{d}(n)] \\
& \cdot v(n)=w^{\prime}(n)+\mu^{\prime}[c(n)-\widehat{c}(n)] v(n) \\
& +\mu^{\prime}[y(n)-\widehat{y}(n)] v(n)+\mu^{\prime}[d(n)-\widehat{d}(n)] v(n) .
\end{aligned}
$$

In general, the physical characteristics of the primary path $P(z)$ are relatively stable in vibration isolation system; if the offline identification accuracy is higher enough and the frequency band is wider enough as well, the offline estimated primary path $\widehat{P}(z)$ will be infinitely close to the primary path $P(z)$, and the online estimated secondary path $\widehat{S}(z)$ will also be infinitely approaching the secondary path $S(z)$; that is to say, $d(n)-\widehat{d}(n) \longrightarrow 0$ and $y(n)-\widehat{y}(n) \longrightarrow 0$. Therefore, this improvement can almost completely restore the signal $v(n)$, which will greatly speed up convergence rate and improve its adaptive control effect.

4.3. Internal Feedback: FFxlms. In Figures 4 and 5, the reference signal $x(n)$ is often selected from the acceleration or exciting force of vibration source, which can interactively generate active control force to suppress vibration by controller, power amplifiers, and actuators. The interaction force, between the upper and middle layers, has an effect on the upper vibration and the middle platform at the same time. However, the algorithms shown in Figures 5, 6, and 8 only consider the force influence on the secondary path and ignore its impact on the reference signal $x(n)$, which may lead to the 


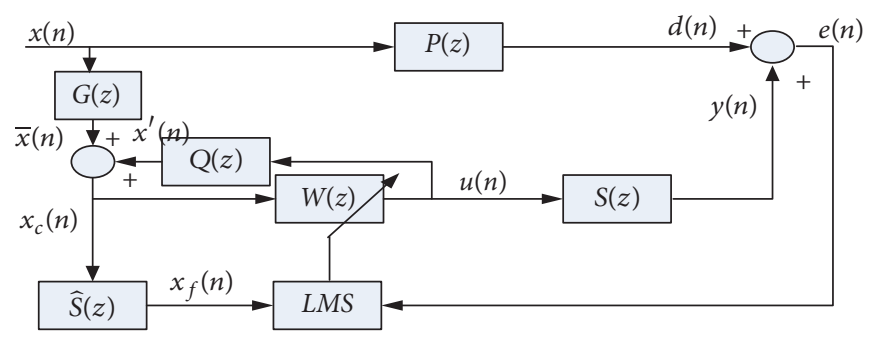

FIGURE 9: Adaptive filtering algorithm structure with internal feedback.

instability of the reference signal and affect convergence rate and accuracy [34].

The transfer function, between the active control force and the reference signal, is called the internal feedback path. Actually, the algorithm shown in Figure 5 is a feedforward control and has faster response speed. In order to improve the steady-state control effect, it must also be considered that the internal feedback has an important influence on the reference signal. The algorithm improved by introducing the internal feedback is shown in Figure 9. According to the system in Figure 5, $x(n)$ can be clearly defined as the exciting force, $\bar{x}(n)$ and $x^{\prime}(n)$ are the acceleration signals generated by the exciting force and active control force, respectively, and $x_{c}(n)$ is the superposed acceleration signal; it can be represented as

$$
x_{c}(n)=\bar{x}(n)+x^{\prime}(n) \text {. }
$$

If $G(n), Q(n)$ can be replaced by FIR filters as follows:

$$
\begin{aligned}
G(n) & =\left[\begin{array}{llll}
g_{0}(n) & g_{1}(n) & \ldots & g_{M-1}(n)
\end{array}\right]^{T} \\
Q(n) & =\left[\begin{array}{llll}
q_{0}(n) & q_{1}(n) & \ldots & q_{M-1}(n)
\end{array}\right]^{T},
\end{aligned}
$$

the acceleration signals $\bar{x}(n), x^{\prime}(n)$ can be expressed as

$$
\begin{aligned}
\bar{x}(n) & =\sum_{i=0}^{M-1} g_{i}(n) x(n-i)=G(n)^{T} X(n) \\
& =X(n)^{T} G(n) \\
x^{\prime}(n) & =\sum_{i=0}^{M-1} q_{i}(n) u(n-i)=Q(n)^{T} U(n) \\
& =U(n)^{T} Q(n)
\end{aligned}
$$

where $X(n), U(n)$ are $M \times 1$ order time series of the external excitation and filter $W(z)$ output, respectively.

$$
\begin{aligned}
& X(n)=\left[\begin{array}{lll}
x(n) & x(n-1) \ldots x(n-M+1)
\end{array}\right]^{T} \\
& U(n)=\left[\begin{array}{lll}
u(n) & u(n-1) \ldots u(n-M+1)
\end{array}\right]^{T}
\end{aligned}
$$

In this case, the reference signal may be expressed as

$$
x_{c}(n)=\sum_{i=0}^{M-1} g_{i}(n) x(n-i)+\sum_{i=0}^{M-1} q_{i}(n) u(n-i)
$$

where $X_{c}(n), \bar{X}(n)$, and $W(n)$ denote the sampling sequence of the superposition signal, the reference sampling sequence, and the filter weight vector, respectively.

$$
\begin{aligned}
X_{c}(n) & =\left[\begin{array}{ll}
x_{c}(n) & x_{c}(n-1) \ldots x_{c}(n-(N-1))
\end{array}\right]^{T} \\
\bar{X}(n) & =\left[\begin{array}{ll}
\bar{x}(n) & \bar{x}(n-1) \ldots \bar{x}(n-(N-1))
\end{array}\right]^{T} \\
W(n) & =\left[\begin{array}{lll}
w_{0}(n) & w_{1}(n) \ldots w_{N-1}(n)
\end{array}\right]^{T}
\end{aligned}
$$

The output $u(n)$ of $N$-order FIR filter can be described as

$$
u(n)=\sum_{i=0}^{N-1} w_{i}(n) x_{c}(n-i)
$$

Substituting (50) and (51) into (52), we can get

$$
\begin{aligned}
U(n) & =W^{T}\left(\text { n) } X_{c}(n)\right. \\
& =W^{T}(\mathrm{n})\left(G(n)^{T} X(n)+Q(n)^{T} U(n)\right) .
\end{aligned}
$$

The controller output can be obtained by introducing the internal feedback path:

$$
U(n)=\frac{W^{T}(\mathrm{n}) G(n)^{T}}{\left(1-W^{T}(\mathrm{n}) Q(n)^{T}\right)} X(n)
$$

where the feedback path corrects the filter $W(z)$ to $W^{\prime}(z)=$ $W^{T}(\mathrm{n}) G(n)^{T} /\left(1-W^{T}(\mathrm{n}) Q(n)^{T}\right)$; when $G(n)=1$ and $Q(n)=$ $0, W^{\prime}(z)$ will degenerate into the Fxlms algorithm without the feedback path shown in Figure 3. Consequently, the improved algorithm has a certain feedback ability and forms the feedforward and feedback control method, which makes the algorithm have faster convergence speed and better steady-state control effect.

4.4. The Hybrid Improved Algorithm: FOFxlms. The shortcomings and improvements of Fxlms algorithm are analyzed in Sections 4.1-4.3, and with the combination of these factors, a novel hybrid improved algorithm, FOFxlms, is proposed in this section, and its structure is illustrated in Figure 10.

The algorithm can realize the secondary path online identification, variable step-size, feedforward and feedback synchronously, and suppression of nonlinear distortion and further extend the applications of Fxlms algorithm in active vibration control as well. In order to verify the hybrid 


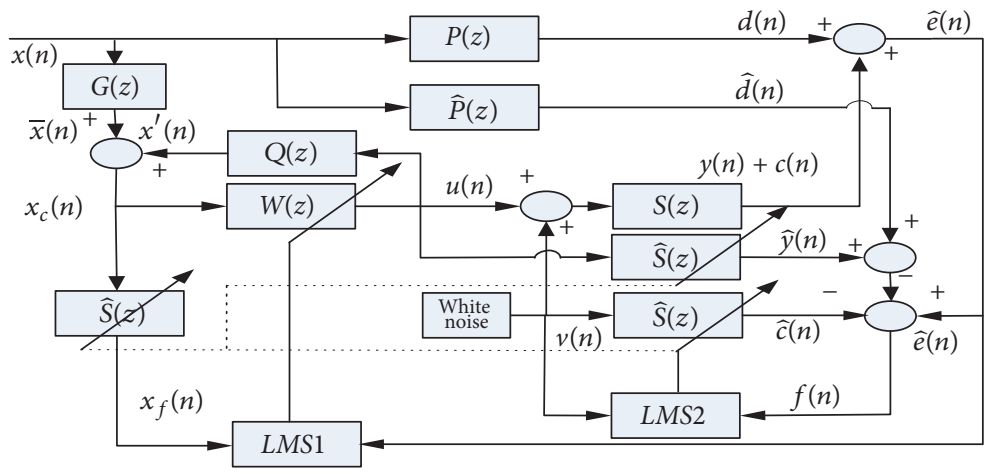

FIGURE 10: The structure of hybrid improved algorithm.

TABLE 1: Double-layer isolation platform parameters.

\begin{tabular}{lccc}
\hline Component & Quality $(\mathrm{M})$ & Stiffness $(\mathrm{K})$ & Damping $(C)$ \\
\hline Upper equipment & $155.5 \mathrm{~kg}$ & $57016.2 \mathrm{~N} / \mathrm{m}$ & $302.7 \mathrm{Ns} / \mathrm{m}$ \\
Middle platform & $22.5 \mathrm{~kg}$ & $190000.1 \mathrm{~N} / \mathrm{m}$ & $290.5 \mathrm{Ns} / \mathrm{m}$ \\
\hline
\end{tabular}

improved algorithm, based on the principle of vibration isolation system in Figure 4, the algorithm effectiveness and control effect are simulated and verified by co-simulation with Adams and Matlab/Simulink.

\section{Numerical Simulations}

In Figures 1 and 2, the equipment speed is $(1450 \pm 50) \mathrm{r} / \mathrm{min}$ with the fundamental frequency $23.3 \mathrm{~Hz}-25.0 \mathrm{~Hz}$ (illustrated with $24.1 \mathrm{~Hz}$ ), and the excitation signal is mainly composed of the fundamental frequency, the second and the third ones in practice, and can be called the multifrequency excitation. The key parameters are listed in Table 1 , and the resonant frequencies are $2.84 \mathrm{~Hz}$ and $15.68 \mathrm{~Hz}$, respectively. The middle acceleration and active control force are taken as the evaluation index.

5.1. Convergence Analysis. According to the algorithms in Section 4, all improved algorithms are evolved by LMS algorithm, so some parameters have similar effect on the convergence performance. In the case of ensuring the system convergence, the influence on convergence characteristics, shown in Figures 11-20, will be studied with the step-size, the signal delay, the secondary path order, and its mutation, respectively.

Assume that the secondary path changed from $S_{(s)}$ to $0.7 S_{(\mathrm{s})}$ at 8 seconds, the offline secondary path does not change at this time, while the online will track this suddenly at the same time. The control effects are as shown in Figures 15-16 with $\mu=0.04$ and 300-order secondary path.

Take the filtered-x adaptive algorithm with feedback path and online identification (FOFxlms); for example, Figures 1112 show the control effect with 300-order secondary path, and Figures 13-14 exhibit the control effect with 32- and 300order secondary path when $\mu=0.04$. In the case of ensuring system convergence, the increase of step-size can improve

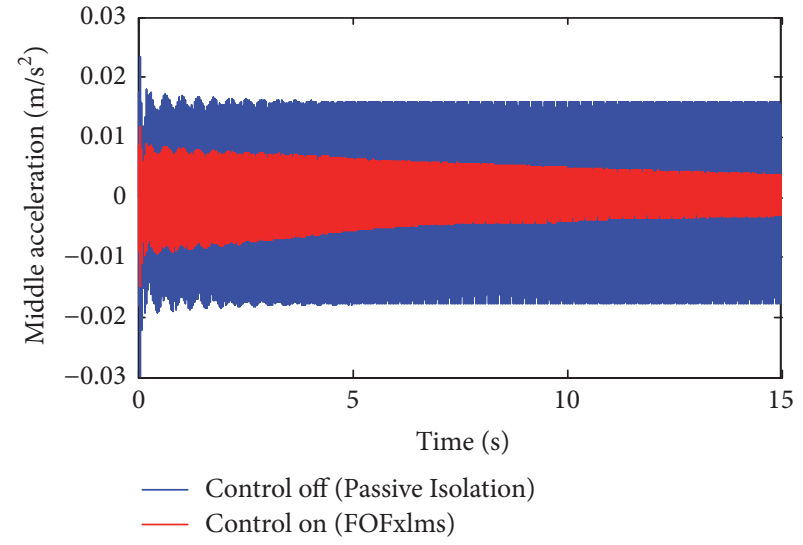

FIGURE 11: Active control time course with $\mu=0.005$ (FOFxlms).

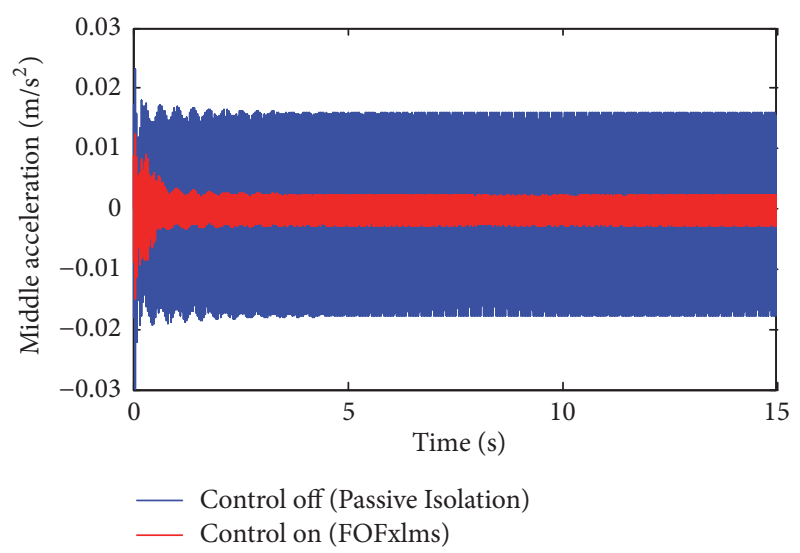

FIGURE 12: Active control time course with $\mu=0.05$ (FOFxlms).

the convergence speed and control effect. And on the basis of guaranteeing real-time operation, adding the secondary path order can also broaden control frequency band, improve the control accuracy, and accelerate the convergence speed, which is consistent with the LMS and its improved algorithms. In addition, the residual error of controlled system is less than the passive vibration isolation, and it shows that all the active control algorithms are indeed effective as well. 


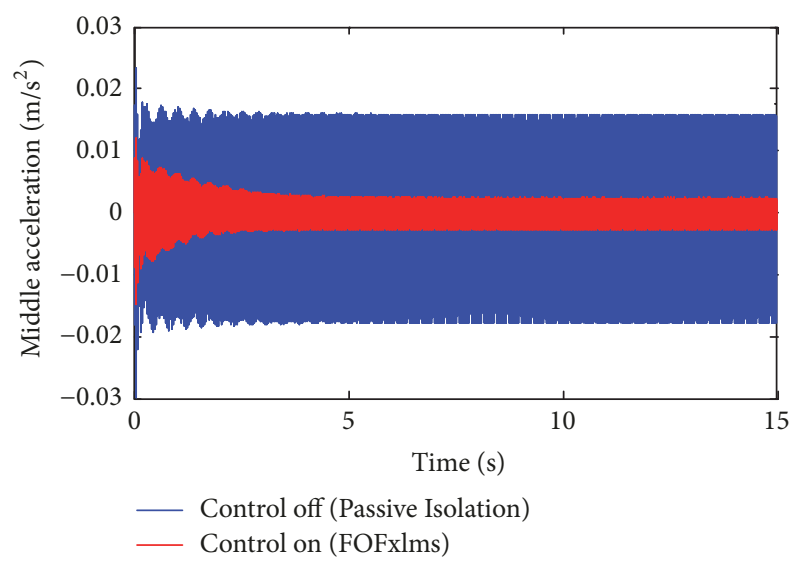

FIGURE 13: Middle acceleration time course with 32-order secondary path (FOFxlms).

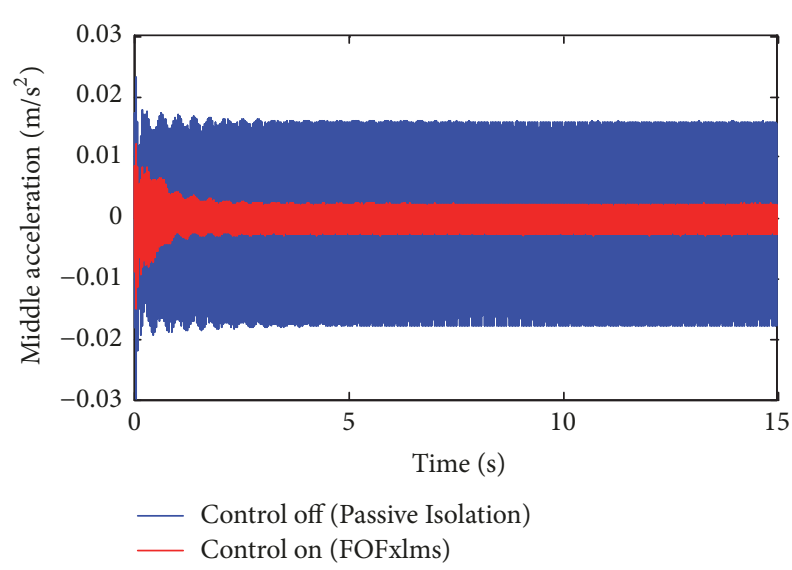

FIgURE 14: Middle acceleration time course with 300-order secondary path (FOFxlms).

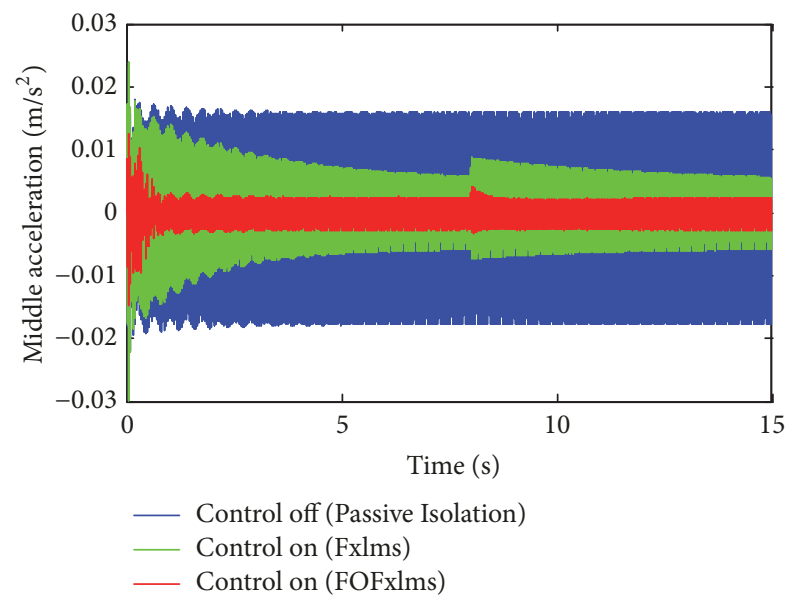

FIGURE 15: Middle acceleration time course with the secondary path mutation (Fxlms and FOFxlms).

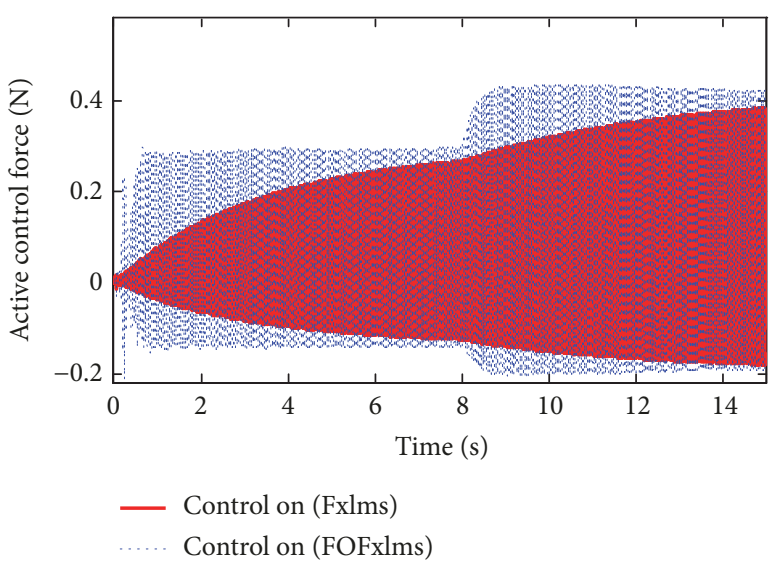

Figure 16: Active control time course with the secondary path mutation (Fxlms and FOFxlms).

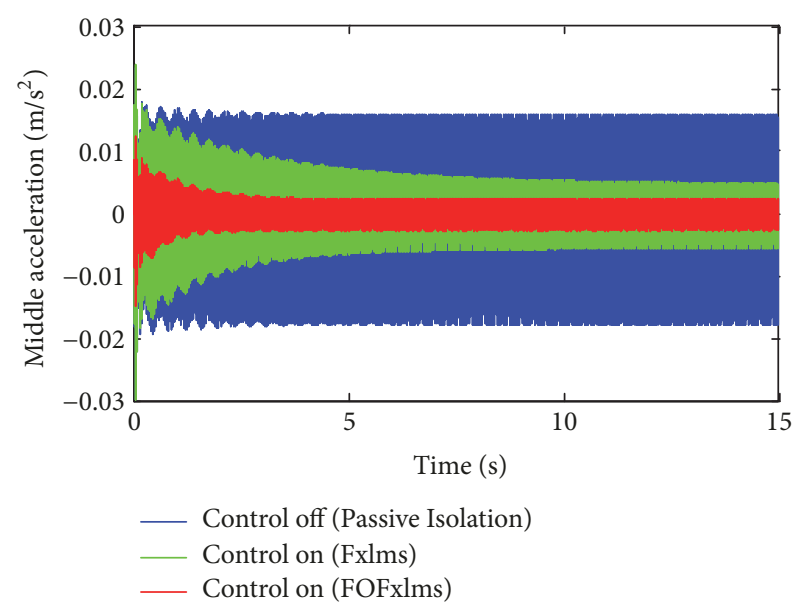

FIGURE 17: Middle acceleration time course with $0.001 \mathrm{~ms}$ time delay (Fxlms and FOFxlms).

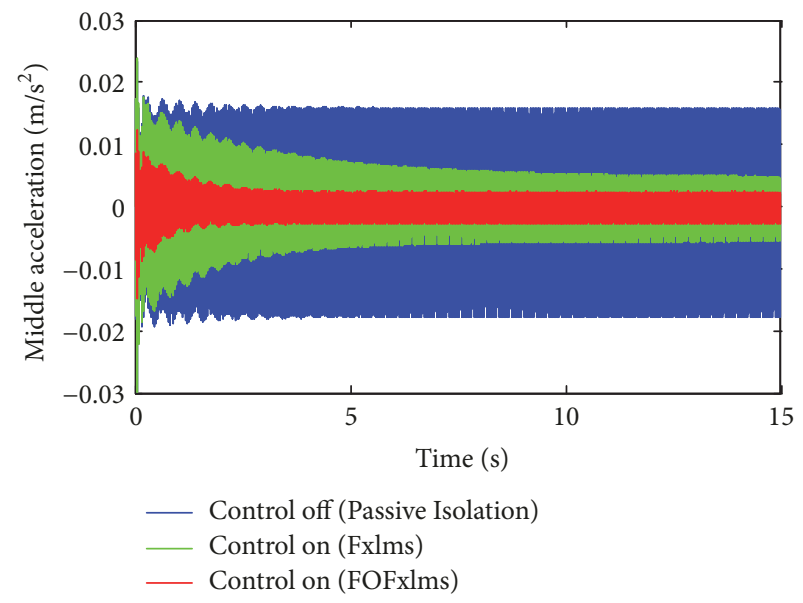

Figure 18: Middle acceleration time course with $0.1 \mathrm{~ms}$ time delay (Fxlms and FOFxlms). 


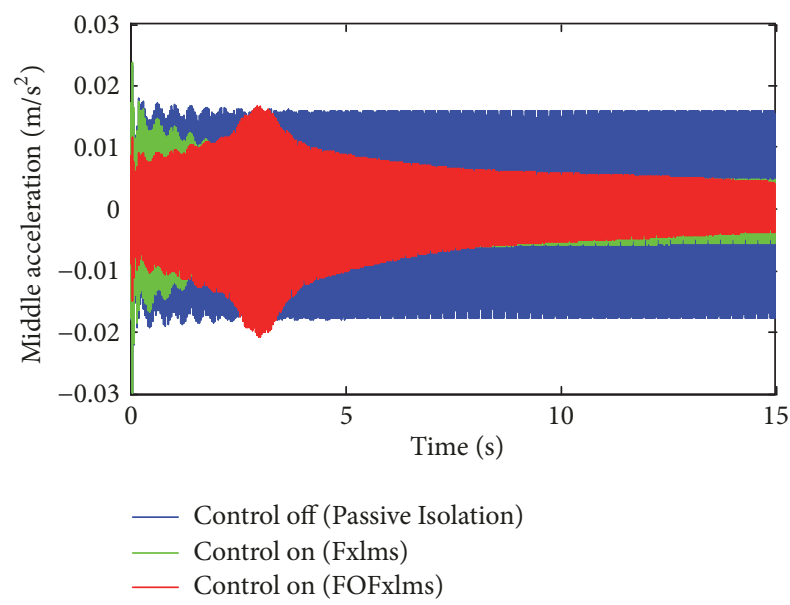

Figure 19: Middle acceleration time course with 1ms time delay (Fxlms and FOFxlms).

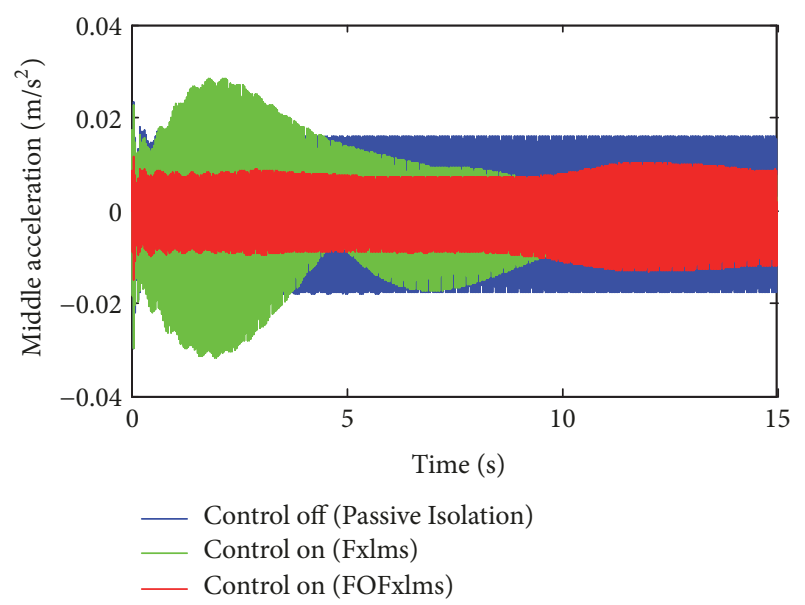

Figure 20: Middle acceleration time course with $10 \mathrm{~ms}$ time delay (Fxlms and FOFxlms).

Figures 15-16 examine the Fxlms and FOFxlms algorithm effects on the offline and online identification with the secondary path mutations, and it can be seen that the Fxlms can only adjust filter $W(z)$ to achieve the goal of the function $J(n) \longrightarrow 0$, while the FOFxlms can dynamically track the change of the secondary path $S_{(\mathrm{s})}$ and quickly adjust active control force to suppress the mutation, which means that the online algorithm can synchronously adjust filter $W^{\prime}(z)$ and $\widehat{S}_{(\mathrm{s})}$ to achieve the goal of the function $J(n) \longrightarrow 0$. Therefore, the improved algorithm has stronger robustness and better real-time.

The Fxlms and FOFxlms control effects in Figures 17-20 are shown with residual signal delay in $0.001 \mathrm{~ms}, 0.1 \mathrm{~ms}, 1 \mathrm{~ms}$, and $10 \mathrm{~ms}$. The delay has direct influence on the residual signal and may cause the deterioration. If the delay is larger enough, maybe the control algorithm will diverge. The former cannot adjust the estimated secondary path, and its steady performance is relatively poorer, while the FOFxlms has better adaptability and stronger stability, and there is no divergence trend when the delay reaches $10 \mathrm{~ms}$.

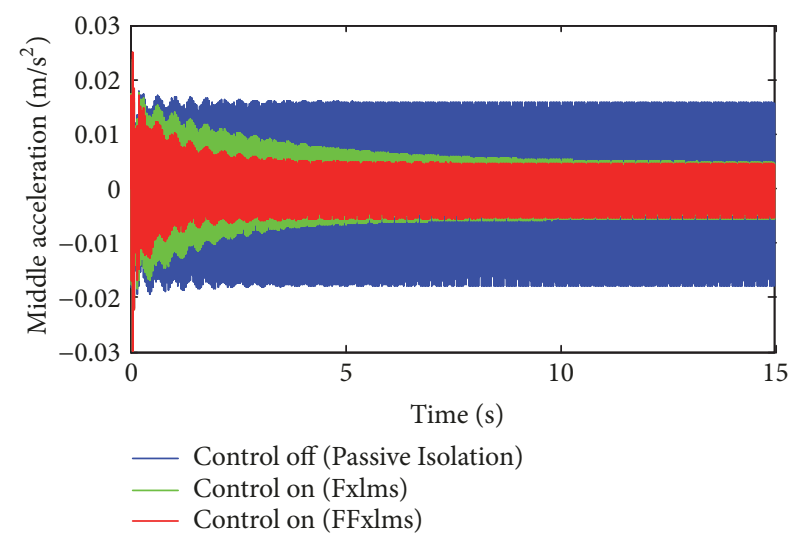

(a) Middle acceleration time course

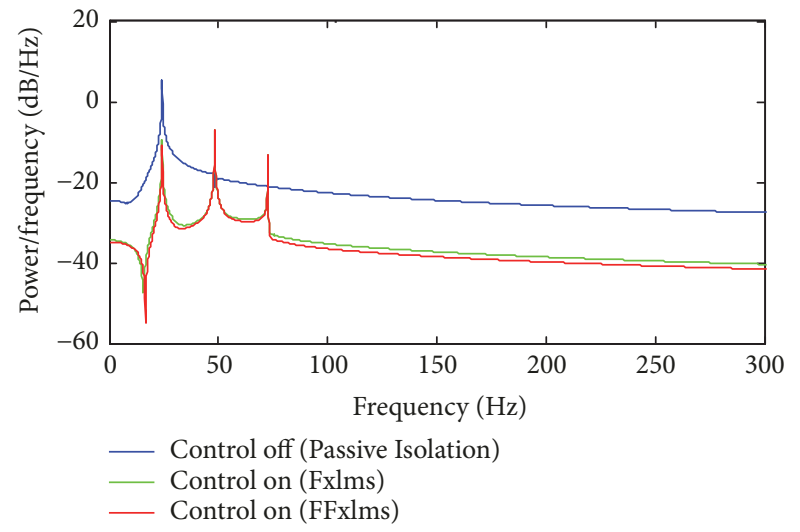

(b) Middle acceleration power spectrum

FIGURE 21: Middle acceleration contrast with and without feedback path (Fxlms and FFxlms).

5.2. Control Effect Analysis. Last, let's analyze convergence of the secondary path online identification; (55) can be used to evaluate online identification performance, where $s_{i}(n), \widehat{s}_{i}(n)$ represent the estimated weighting coefficients of the offline and online secondary path, respectively.

$$
\Delta S(d B)=10 \log _{10}\left\{\frac{\sum_{i=0}^{N_{2}}\left(s_{i}(n)-\widehat{s}_{i}(n)\right)^{2}}{\sum_{i=0}^{N_{2}}\left[s_{i}(n)\right]^{2}}\right\}
$$

In Figures 21-24, the residual error and active control force are compared in time and frequency domains for the passive vibration isolation, the Fxlms, the FFxlms, and the FOFxlms, respectively. And the performance indicators of line spectrum control are given by a quantitative description in Table 2 and Figures 25-26. Three algorithms, including Fxlms, FFxlms, and FOFxlms, have a certain control effect in time domain, and the convergence speed and control effect of residual signal and active control force are enhanced in turn. The peak line spectrum of residual signal is reduced by $14.99 \mathrm{~dB} /-9.66 \mathrm{~dB} /-7.53 \mathrm{~dB}, 16.50 \mathrm{~dB} /-9.78 \mathrm{~dB} /-7.52 \mathrm{~dB}$, and $23.39 \mathrm{~dB} /-4.00 \mathrm{~dB} /-1.78 \mathrm{~dB}$ in frequency domain, respectively. And the control effects of the algorithms mentioned above were heightened for the fundamental frequency in turn, while the deficiency is the deterioration of harmonic control effect in Fxlms and FFxlms algorithms. 
TABLE 2: Control effect comparison on several control algorithms.

\begin{tabular}{|c|c|c|c|c|c|c|c|c|c|c|}
\hline & \multicolumn{4}{|c|}{ Middle acceleration or residual error $(\mathrm{dB})$} & \multicolumn{3}{|c|}{ Vibration attenuation $(\mathrm{dB})$} & \multicolumn{3}{|c|}{ Active control force $(\mathrm{dB})$} \\
\hline & Passive & Fxlms & FFxlms & FOFxlms & Fxlms & FFxlms & FOFxlms & Fxlms & FFxlms & FOFxlms \\
\hline Fundamental frequency & 5.48 & -9.51 & -11.02 & -17.91 & 14.99 & 16.50 & 23.39 & 25.52 & 25.81 & 25.96 \\
\hline Double frequency & -16.78 & -7.12 & -7.00 & -12.78 & -9.66 & -9.78 & -4.00 & 20.61 & 20.45 & 20.75 \\
\hline Triple frequency & -20.91 & -13.38 & -13.39 & -19.13 & -7.53 & -7.52 & -1.78 & 13.10 & 13.15 & 13.34 \\
\hline
\end{tabular}

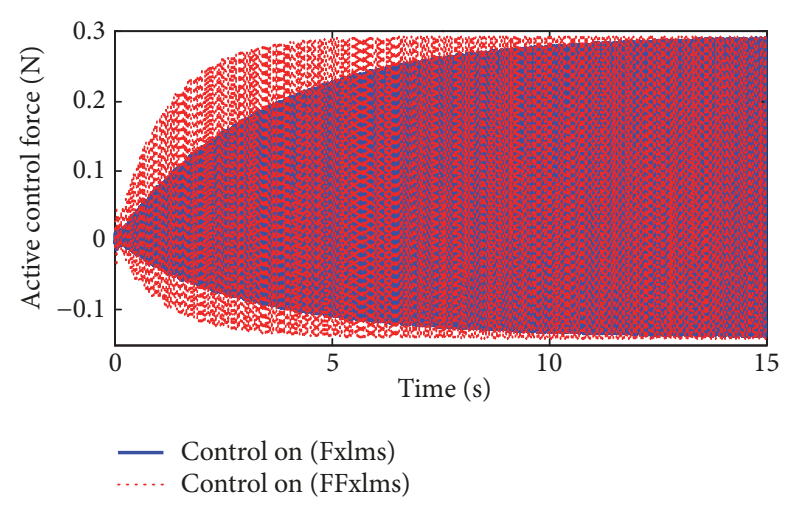

(a) Active control time course

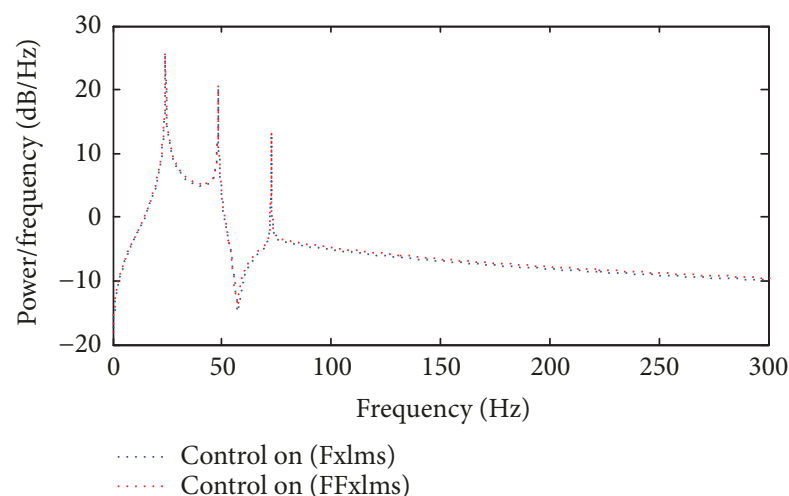

(b) Active control power spectrum

FIgURE 22: Active control contrast with and without feedback path (Fxlms and FFxlms).

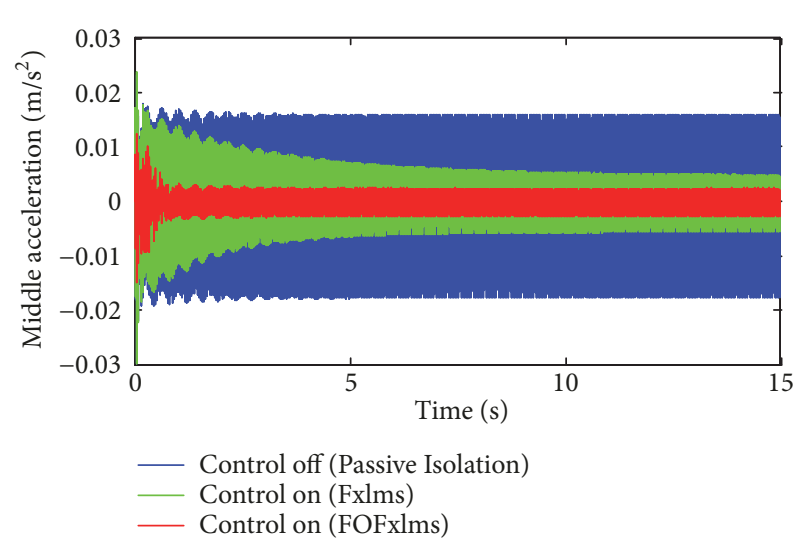

(a) Middle acceleration time course

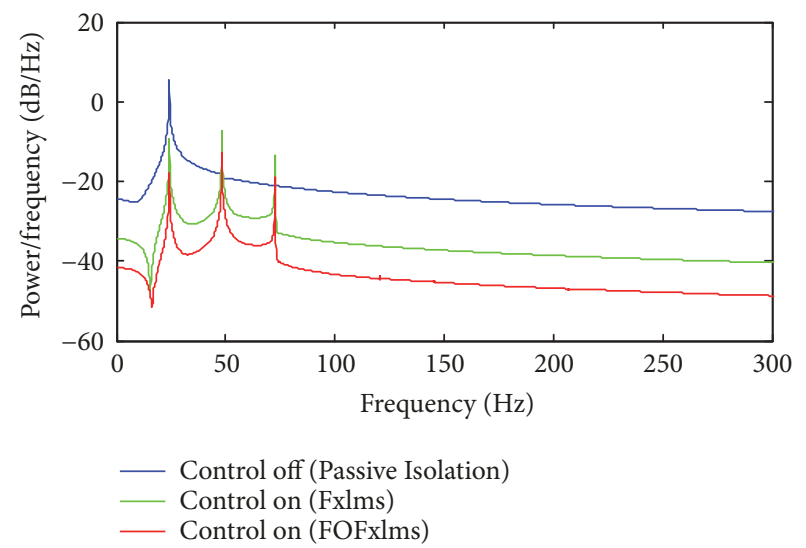

(b) Middle acceleration power spectrum

FIGURE 23: Middle acceleration contrast with and without feedback path and online identification (Fxlms and FOFxlms).

Actually, the harmonic frequency is bigger than the fundamental one and amplitude is smaller, and its transmission attenuation is much faster, so we mainly focus on the fundamental frequency vibration, because it can transmit much farther. In terms of time domain (Figures 21(a)-24(a)), the algorithm proposed in the paper has better control effect. On the other hand, the harmonic control effect is not as ideal as the fundamental one in Figures 21(b)-24(b), which is mainly related to the control filter order, it is because the order cannot be large enough to describe the model properties, and the hybrid algorithm has been improved to a certain extent. In addition, for the multifrequency excitation, the active control force steady amplitude of three algorithms is basically equivalent, which shows that no matter what kind of method to suppress the same external incentives, it needs to consume the same energy of secondary vibration source as well.

In addition, from Figure 27, it can be seen that the evaluation function $\Delta S(d B)$ will achieve the minimum and tend to be stable after 7 seconds, and then the weight coefficient will be a convergence state, which indicates that the improved method with online identification is correct and effective.

\section{Conclusions}

A novel hybrid FOFxlms algorithm is proposed in this paper, which improves the traditional Fxlms performance by introducing the nonlinear distortion, the variable stepsize, the internal feedback, and the online identification. A dynamic model of double-layer vibration isolation model is 


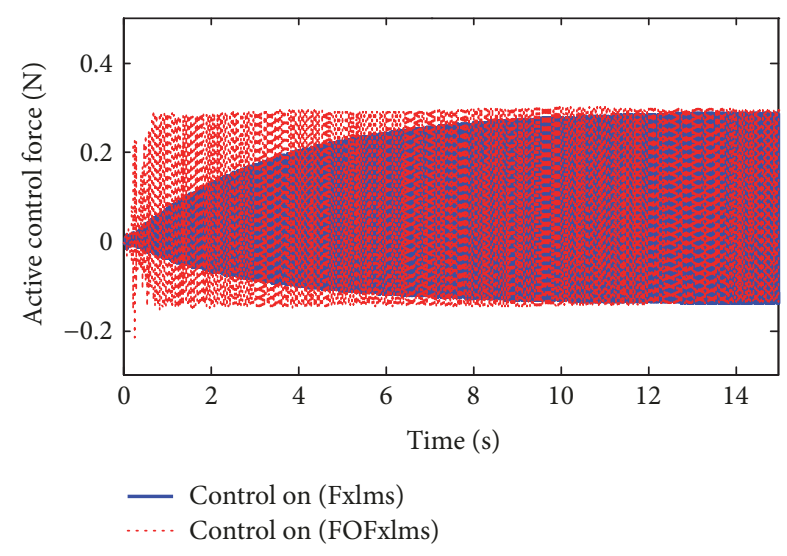

(a) Active control time course

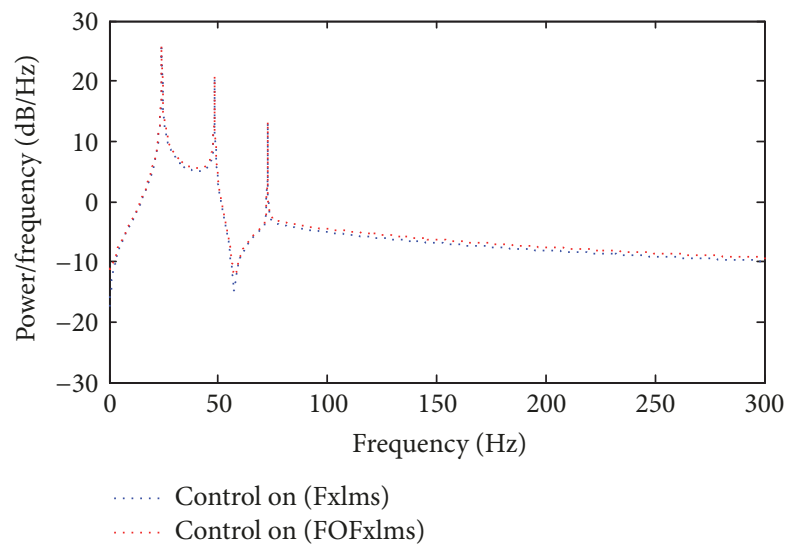

(b) Active control power spectrum

FIGURE 24: Active control contrast with and without feedback path and online identification (Fxlms and FOFxlms).

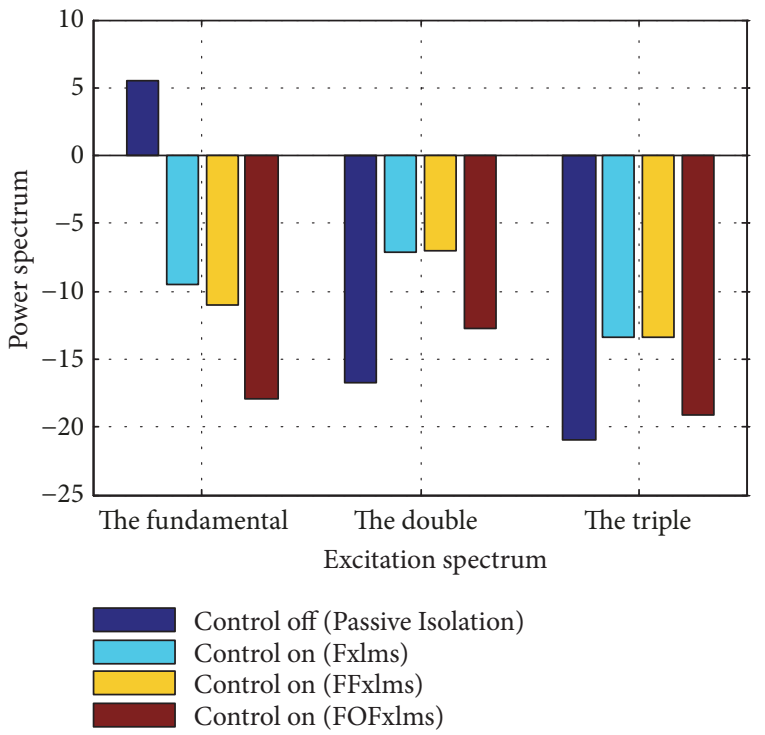

Figure 25: Control effect comparison with several control algorithms (the residual error).

established as well, and its dynamic equations are also carried out to obtain the mathematical model of all physical paths. Then, the algorithm convergence and control effect presented in the paper are validated and analyzed by co-simulation with Adams and Matlab/Simulink.

The results demonstrate that the parameters, including an reasonable step, a secondary path order, and time delay, have important influence on the convergence speed and control effect. The online identification can effectively identify the time-varying characteristics of the secondary path, and the internal feedback can compensate for the steady-state control effect, while the variable step-size by introducing residual error can improve the system convergence characteristics. Compared with the passive vibration isolation, three algorithms have better control effect in time domain; all of

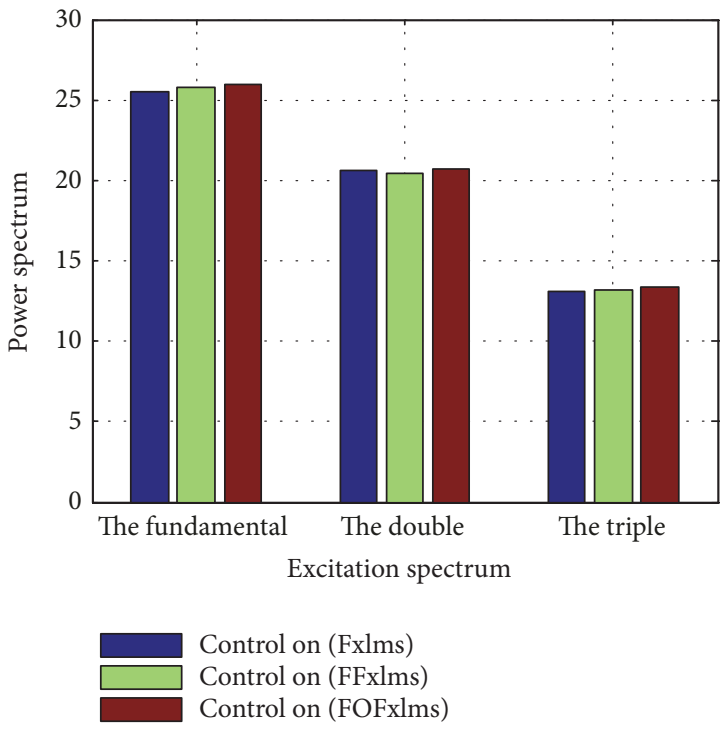

FIGURE 26: Control effect comparison with several control algorithms (the active control force).

them can effectively reduce the residual error, increase the response speed, and enhance the control effect in turn. They also have better ability to control fundamental frequency, and the Fxlms control effect of harmonic line spectrum is basically equal to the FFxlms, but the FOFxlms is relatively enhanced by the hybrid improvement, which indicates that the proposed multi-related improvement is correct and effective. And several methods basically require the same steadystate active control force, which also shows that it needs to consume the same secondary energy to suppress the same amount of external excitation.

\section{Conflicts of Interest}

The authors declare that there are no conflicts of interest regarding the publication of this paper. 


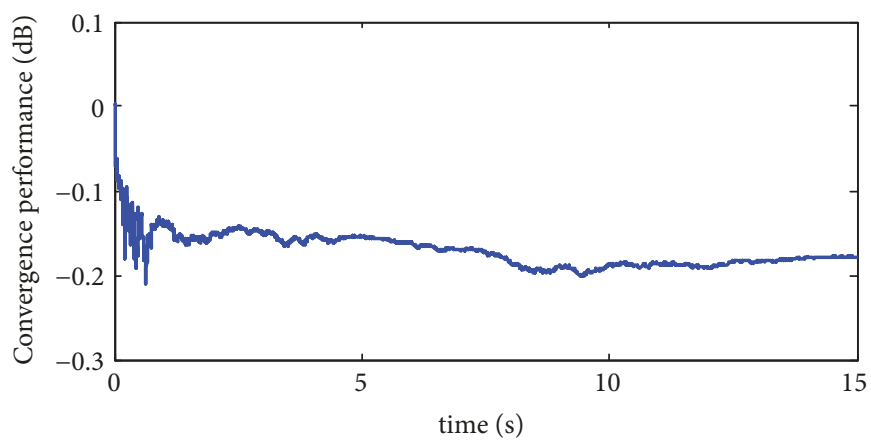

FIGURE 27: $\Delta S(d B)$ convergence of secondary path with online identification.

\section{Acknowledgments}

This work was supported by State Key Laboratory of Ocean Engineering (Shanghai Jiao Tong University) (Grant no. 1714) and National Natural Science Funds of China (51579242, 51509253).

\section{References}

[1] J. Niu, K. Song, and C. W. Lim, "On active vibration isolation of floating raft system," Journal of Sound and Vibration, vol. 285, no. 1-2, pp. 391-406, 2005.

[2] L. He and W. Xu, "Naval vessel machinery mounting technology and its recent advances," Acta Acustica united with Acustica, vol. 38, no. 2, pp. 128-134, 2013.

[3] W. John, Sensor and Actuator Networks for Acoustic Signature Monitoring and Control, Undersea Defence Technology, Nice, France, 1999.

[4] M. Winberg, S. Johansson, L. Håkansson, I. Claesson, and T. Lagö, "Active vibration isolation in ships: A pre-analysis of sound and vibration problems," International Journal of Acoustics and Vibration, vol. 10, no. 4, pp. 175-196, 2005.

[5] "New technologies-active isolation, Home page of paulstravibrachoc Inc," http://www.Paulstra-vibrachoc.com/Active isolatioll_GB.pdf.

[6] S. Daley and I. Zazas, "A recursive least squares based control algorithm for the suppression of tonal disturbances," Journal of Sound and Vibration, vol. 331, no. 6, pp. 1270-1290, 2012.

[7] C. D. Petersen, R. Fraanje, B. S. Cazzolato, A. C. Zander, and C. H. Hansen, "A Kalman filter approach to virtual sensing for active noise control," Mechanical Systems and Signal Processing, vol. 22, no. 2, pp. 490-508, 2008.

[8] H.-R. Hao, H.-B. Bai, and H.-J. Zhang, "Robust control of a 6DOF active-passive vibration isolation platform," Zhendong yu Chongji, vol. 31, no. 7, pp. 122-127, 2012.

[9] X.-K. Gao and H. Shao, "Semi-active optimal control of an intelligent damping double-deck vibration isolation system," Zhendong yu Chongji, vol. 31, no. 19, pp. 128-133, 2012.

[10] C. H. Hansen, D. S. Scott, X. J. Qiu et al., Active Control of Noise and Vibration, Taylor\& Francis, Boca Raton, FL, USA, 2013.

[11] S. Carra, M. Amabili, R. Ohayon, and P. M. Hutin, "Active vibration control of a thin rectangular plate in air or in contact with water in presence of tonal primary disturbance," Aerospace Science and Technology, vol. 12, no. 1, pp. 54-61, 2008.

[12] K. Ma, "Vibration control of smart structures with bonded PZT patches: Novel adaptive filtering algorithm and hybrid control scheme," Smart Materials and Structures, vol. 12, no. 3, pp. 473482, 2003.

[13] J. J. Carnahan and C. M. Richards, "A modification to filteredX LMS control for airfoil vibration and flutter suppression," Journal of Vibration and Control, vol. 14, no. 6, pp. 831-848, 2008.

[14] X. Zhu, Z. Gao, Q. Huang, S. Gao, and E. Jiang, "Analysis and implementation of MIMO FULMS algorithm for active vibration control," Transactions of the Institute of Measurement and Control, vol. 34, no. 7, pp. 815-828, 2012.

[15] J. Zheng, J. Cheng, Y. Nie, and S. Luo, "A signal processing method for fault diagnosis-complete ensemble local characteristic-scale decomposition," Journal of Vibration Engineering, vol. 27, no. 4, pp. 637-646, 2014.

[16] D. P. Das, D. J. Moreau, and B. S. Cazzolato, "A computationally efficient frequency-domain filtered-X LMS algorithm for virtual microphone," Mechanical Systems and Signal Processing, vol. 37, no. 1-2, pp. 440-454, 2013.

[17] Q. Huang, J. Luo, H. Li, and X. Wang, "Analysis and implementation of a structural vibration control algorithm based on an IIR adaptive filter," Smart Materials and Structures, vol. 22, no. 8, Article ID 085008, 2013.

[18] H.-W. Kim, H.-S. Park, S.-K. Lee, and K. Shin, "Modifiedfiltered-u LMS algorithm for active noise control and its application to a short acoustic duct," Mechanical Systems and Signal Processing, vol. 25, no. 1, pp. 475-484, 2011.

[19] S.-W. Ning and Z.-Y. Shi, "QR-decomposition-based least squares lattice adaptive filter algorithms for active noise control," Zhendong Gongcheng Xuebao, vol. 26, no. 3, pp. 363-373, 2013.

[20] Z. Yulin and Z. Xiuyang, "Wavelet adaptive algorithm and its application to MRE noise control system," Shock and Vibration, vol. 2015, Article ID 968082, 2015.

[21] M. T. Akhtar, M. Abe, and M. Kawamata, "A new variable step size LMS algorithm-based method for improved online secondary path modeling in active noise control systems," IEEE Transactions on Audio, Speech and Language Processing, vol. 14, no. 2, pp. 720-726, 2006.

[22] A. Carini and S. Malatini, "Optimal variable step-size nlms algorithms with auxiliary noise power scheduling for feedforward active noise control," IEEE Transactions on Audio, Speech and Language Processing, vol. 16, no. 8, pp. 1383-1395, 2008.

[23] S.-C. Chan and Y. Chu, "Performance analysis and design of FxLMS algorithm in broadband ANC system with online secondary-path modeling," IEEE Transactions on Audio, Speech and Language Processing, vol. 20, no. 3, pp. 982-993, 2012. 
[24] Y. X. Pu, F. Zhang, and J. H. Jiang, "Active vibration control with a new online secondary path modeling method, Journal of Vibration," Journal of Vibration, Measurement \& Diagnosis, vol. 36, no. 1, pp. 28-35, 2016.

[25] J. G. Avalos, A. Rodriguez, H. M. Martinez, J. C. Sanchez, and H. M. Perez, "Multichannel Filtered-X Error Coded Affine Projection-Like Algorithm with Evolving Order," Shock and Vibration, vol. 2017, Article ID 3864951, 2017.

[26] Y. L. Zhou and Q. Z. Zhang, Active Noise and Vibration Control - Principle, Algorithm and Implementation, Tsinghua University Press, Beijing, China, 2013.

[27] I. Tabatabaei and W. H. Abdulla, "FxLMS-based active noise control a quick review," Asia Pacific Signal and Information Processing Association, pp. 2011-2020, 2011.

[28] B. F. Boroujeny, Adaptive Filtering Theories and Applications, John Wiley \& Sons, Ltd, Chichester, UK, 2nd edition, 2013.

[29] P. S. R. Diniz, Adaptive Filtering: Algorithms and Practical Implementation, Springer, New York, NY, USA, 4th edition, 2013.

[30] B. Pathak and P. P. Hirave, "FXLMS algorithm for feed-forward active noise cancellation," in Proceedings of the Conference Advances in Computer, Electronics and Electrical Engineering, pp. 18-22, 2012.

[31] Y.-Y. Wang, Y.-N. Li, W. Sun, C. Yang, and G.-H. Xu, "FxLMS method for suppressing in-wheel switched reluctance motor vertical force based on vehicle active suspension system," Journal of Control Science and Engineering, vol. 2014, Article ID 486140, 2014.

[32] M. T. Akhtar and W. Mitsuhashi, "A modified normalized FxLMS algorithm for active control of impulsive noise," in Proceedings of the 18th European Signal Processing Conference, EUSIPCO 2010, pp. 1-5, dnk, August 2010.

[33] L. J. Eriksson and M. C. Allie, "Use of random noise for on-line transducer modeling in an adaptive active attenuation system," The Journal of the Acoustical Society of America, vol. 85, no. 2, pp. 797-802, 1989.

[34] C. K. Arthikeyan and S. Prakash, "FxLMS algorithm with feedback neutralization for active vibration control," Journal Institute of Smart Structures and Systems, vol. 1, no. 1, pp. 2333, 2012. 


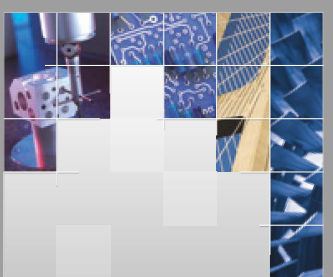

\section{Enfincering}
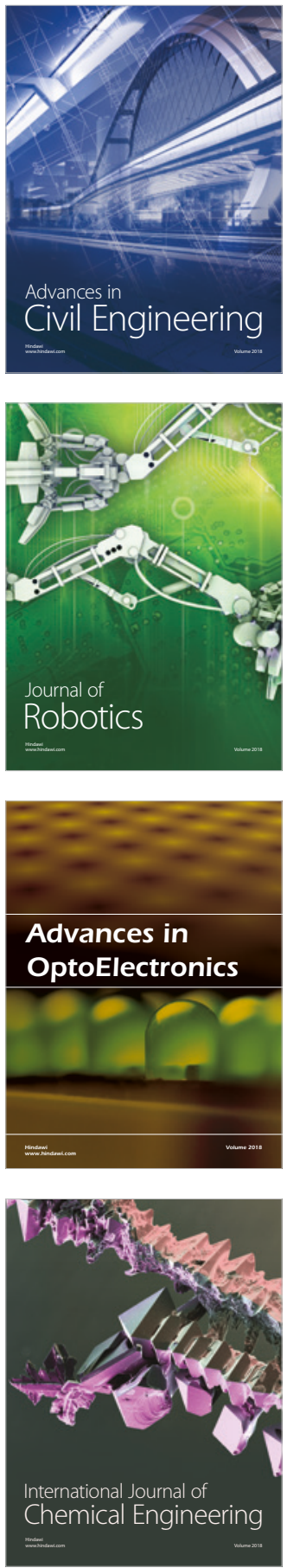

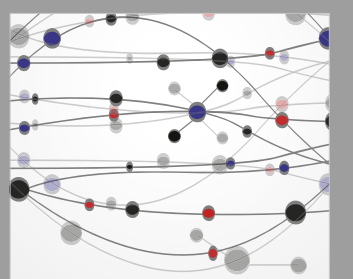

\section{Rotating \\ Machinery}

The Scientific World Journal

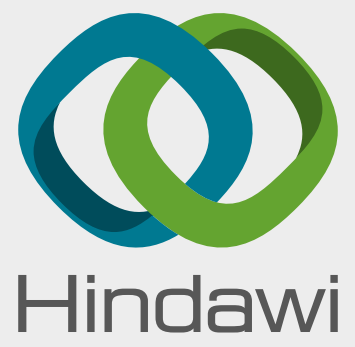

Submit your manuscripts at

www.hindawi.com
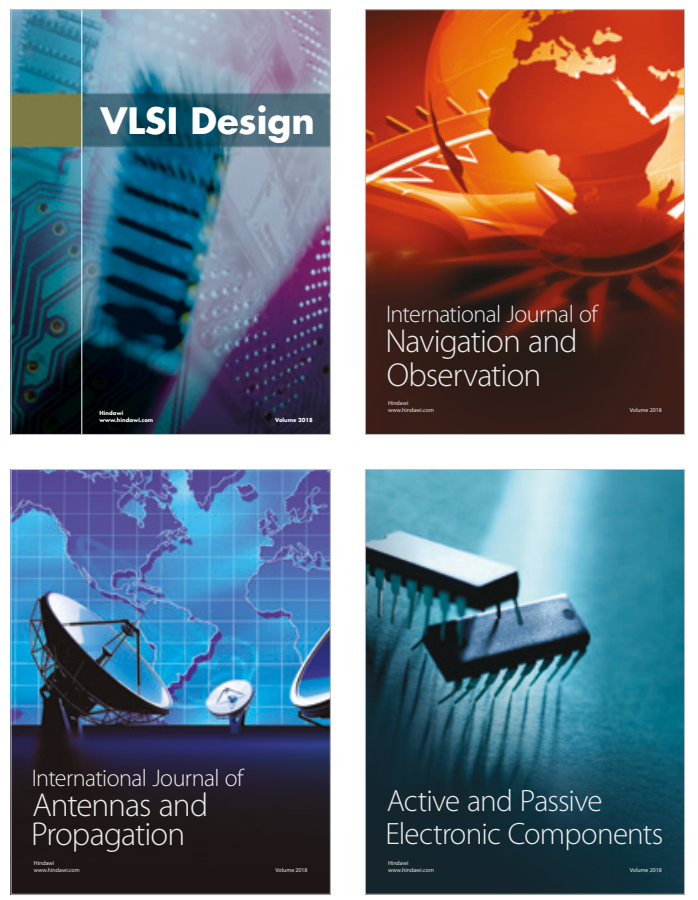
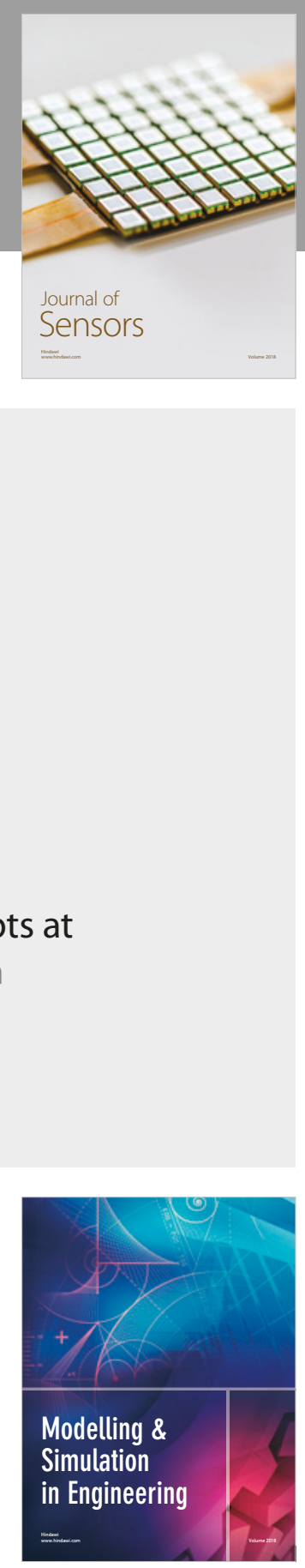

\section{Advances \\ Multimedia}
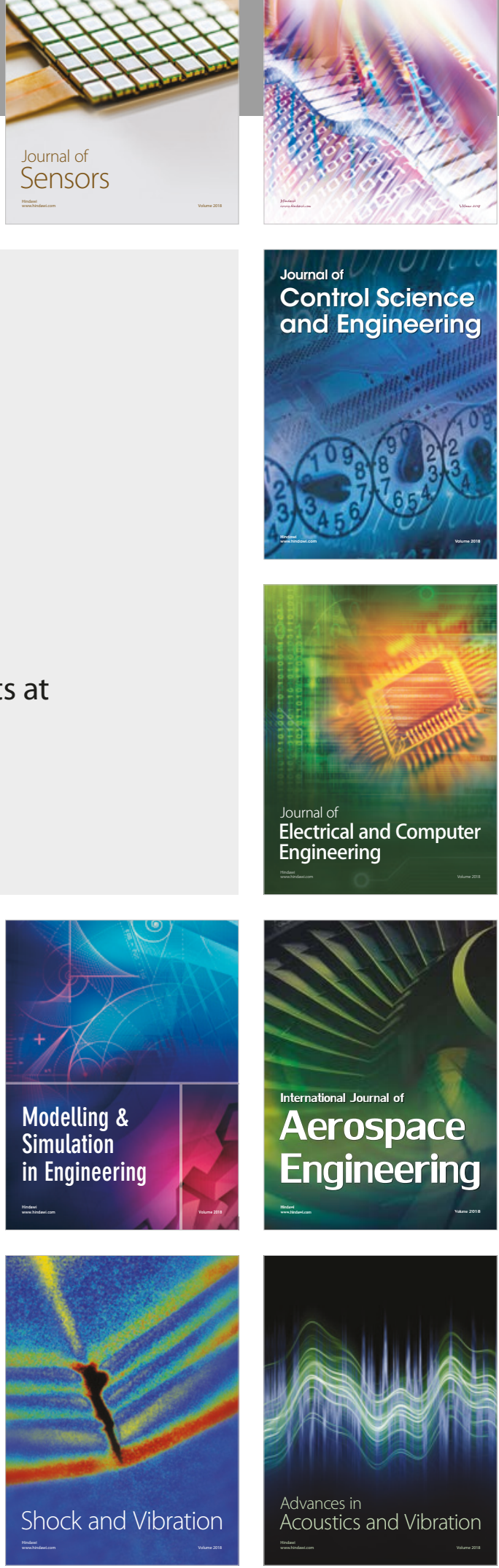